\title{
Impaired left atrial reservoir and conduit strain in patients with atrial fibrillation and extensive left atrial fibrosis
}

\author{
Luuk H. G. A. Hopman, Mark J. Mulder, Anja M. van der Laan, Ahmet Demirkiran, Pranav Bhagirath, \\ Albert C. van Rossum, Cornelis P. Allaart and Marco J. W. Götte* (i)
}

\begin{abstract}
Background: Atrial fibrillation (AF) is associated with profound structural and functional changes in the atria. In the present study, we investigated the association between left atrial (LA) phasic function and the extent of LA fibrosis using advanced cardiovascular magnetic resonance (CMR) imaging techniques, including 3-dimensional (3D) late gadolinium enhancement (LGE) and feature tracking.
\end{abstract}

Methods: Patients with paroxysmal and persistent AF $(n=105)$ underwent CMR in sinus rhythm. LA global reservoir strain, conduit strain and contractile strain were derived from cine CMR images using CMR feature tracking. The extent of LA fibrosis was assessed from 3D LGE images. Healthy subjects underwent CMR and served as controls $(n=19)$.

Results: Significantly lower LA reservoir strain, conduit strain and contractile strain were found in AF patients, as compared to healthy controls $(-15.9 \pm 3.8 \%$ vs. $-21.1 \pm 3.6 \% P<0.001,-8.7 \pm 2.7 \%$ vs. $-12.6 \pm 2.5 \% P<0.001$ and $-7.2 \pm 2.3 \%$ vs. $-8.6 \pm 2.2 \% P=0.02$, respectively). Patients with a high degree of LA fibrosis (dichotomized by the median value) had lower reservoir strain and conduit strain compared to patients with a low degree of $L A$ fibrosis $(-15.0 \pm 3.9 \%$ vs. $-16.9 \pm 3.3 \%, P=0.02$ and $-7.9 \pm 2.7 \%$ vs. $-9.5 \pm 2.6 \%, P=0.01$, respectively). In contrast, no difference was found for LA contractile strain $(-7.1 \pm 2.4 \%$ vs. $-7.4 \pm 2.3 \%, P=0.55)$.

Conclusions: Impaired LA reservoir and conduit strain are present in AF patients with extensive atrial fibrosis. Future studies are needed to examine the biologic nature of this association and possible therapeutic implications.

Keywords: Atrial remodeling, Atrial fibrillation, Atrial strain, Atrial fibrosis, Cardiovascular magnetic resonance (CMR)

\section{Background}

Atrial remodeling is an important hallmark feature of atrial fibrillation (AF) [1]. This process is characterized by inflammation, lipidosis, and fibrosis, leading to changes in atrial structure, geometry, volume, and function [2].

Cardiovascular magnetic resonance (CMR) imaging has emerged as the gold standard method to evaluate the atrial remodeling process. This imaging modality

*Correspondence: mjw.gotte@amsterdamUMC.nl

Department of Cardiology, Amsterdam UMC, Vrije Universiteit Amsterdam, Amsterdam Cardiovascular Sciences, De Boelelaan 1118 , 1081 HV Amsterdam, The Netherlands provides non-invasive assessment of left atrial (LA) wall fibrosis using three-dimensional (3D) late gadolinium enhancement (LGE) imaging [3, 4], as well as data on LA volume, geometry, and function. Assessment of LA function is complex, involving three phases, with important interplay between LA and left ventricular (LV) function in each phase. In systole, the LA serves as a reservoir, involving LA compliance, relaxation, and descent of LV base [5]. During early diastole, LA conduit function involves LV diastolic function (suction force) and LA compliance. At the end of diastole, LA contractile function depends on contractility, LV compliance, and original author(s) and the source, provide a link to the Creative Commons licence, and indicate if changes were made. The images or other third party material in this article are included in the article's Creative Commons licence, unless indicated otherwise in a credit line to the material. If material is not included in the article's Creative Commons licence and your intended use is not permitted by statutory regulation or exceeds the permitted use, you will need to obtain permission directly from the copyright holder. To view a copy of this licence, visit http://creativecommons.org/licenses/by/4.0/. The Creative Commons Public Domain Dedication waiver (http://creativeco mmons.org/publicdomain/zero/1.0/) applies to the data made available in this article, unless otherwise stated in a credit line to the data. 
end-diastolic pressure [6, 7]. CMR feature tracking post processing software has been developed for a more accurate assessment of atrial function in these three phases which can be used to determine the LA reservoir strain, conduit strain, and contractile strain $[8,9]$. Recent studies have shown that LA fibrosis negatively impacts global atrial function $[10,11]$. However, limited data exist on the relation between LA fibrosis and LA phasic strain. In the present study, we investigated the association between LA strain and LA fibrosis during all three phases of the cardiac cycle in AF patients, using advanced CMR imaging and post-processing techniques including 3D LGE and feature tracking.

\section{Methods}

\section{Study design}

This prospective single-center study was conducted in accordance with the Declaration of Helsinki. The study protocol was approved by the local medical ethics committee (Amsterdam UMC, location VU University Medical Center, Amsterdam, The Netherlands). Written informed consent was obtained from all individuals.

\section{Study population}

A total of 105 AF patients were enrolled between July 2018 and April 2021. All patients had paroxysmal or persistent AF according to the European Society of Cardiology/EHRA guidelines $[12,13]$ and were scheduled to undergo a first pulmonary vein isolation (PVI) ablation procedure. Prior to the PVI catheter ablation, patients underwent CMR imaging while in sinus rhythm.

Exclusion criteria were general CMR contraindications (including metal implants and claustrophobia), contraindications for a gadolinium-based contrast agent, mechanical heart valves, a cardiac implantable electronic device, and absence of sinus rhythm as the time of CMR. Nineteen healthy subjects underwent CMR imaging without contrast administration to assess LA volume and function, and served as controls.

\section{CMR protocol}

All scans were performed using a 1.5 T CMR (AVANTO or SOLA, Siemens Healthineers, Erlangen, Germany) and 32-channel array coil. The CMR protocol included balanced steady state free precession cine imaging in long axis orientations (two-chamber and four-chamber view), a $3 \mathrm{D}$ contrast enhanced magnetic resonance angiogram (CE-MRA) and 3D high resolution LGE images. For cine imaging, typical in-plane resolution was $1.3 \times 1.3 \mathrm{~mm}^{2}$ and acquisition parameters were as follows: repetition time, 41-47 ms; echo time, $1.6 \mathrm{~ms}$; slice thickness, $5 \mathrm{~mm}$; flip angle, $60-75^{\circ}$; matrix, $256 \times 208 \mathrm{~mm}$; temporal resolution, $<40 \mathrm{~ms}$.
An electrocardiogram (ECG) gated free-breathing navigator-based 3D CE-MRA of the LA and pulmonary veins was obtained immediately after a $20 \mathrm{~mL}(1 \mathrm{~mL} / \mathrm{sec})$ single dose bolus injection of contrast agent (Dotarem ${ }^{\circledR}$, Guerbet LLC, Roissy, France) followed by a body weight dependent $(0.2 \mathrm{mmol} / \mathrm{kg})$ slow infusion of contrast agent (slow infusion dose; $2.5-30.0 \mathrm{~mL}$, infusion rate; $0.1-$ $0.25 \mathrm{~mL} / \mathrm{sec}$ ). A navigator (5 $\mathrm{mm}$ acceptance window) was positioned on the right hemi-diaphragm to acquire data during the end of respiratory expiration. Typical acquisition parameters were: repetition time/ echo time was $5.5 / 3.0 \mathrm{~ms}$; flip angle, $25^{\circ}$; in-plane resolution was $1.25 \times 1.25 \mathrm{~mm}$ with slice thickness $2.5 \mathrm{~mm}$ (reconstructed to $0.625 \times 0.625 \times 1.25 \mathrm{~mm}$ ).

High resolution 3D LGE images were acquired using a navigator-based respiration- and ECG-gated inversion recovery prepared gradient echo pulse sequence applied approximately $20 \mathrm{~min}$ after contrast injection. The voxel size was $1.25 \times 1.25 \times 2.5 \mathrm{~mm}$ (reconstructed to $0.625 \times 0.625 \times 1.25 \mathrm{~mm}$ ). Other typical sequence parameters were as follows: repetition time/ echo time was 5.2/2.4 ms; flip angle, $20^{\circ}$. Depending on the respiratory pattern and the heart rate of the patient, acquisition of the 3D CE-MRA and LGE series took approximately 10-15 min each.

\section{CMR data analysis \\ $L A$ volume and function}

Cine image analysis was performed using cvi42 (version 5.11, Circle Cardiovascular Imaging, Inc, Calgary, Alberta, Canada). Volumetric data of the LA and LV were calculated from two-chamber and four-chamber cine images using the biplane method. LA volume (LAV) was divided in minimal $\left(\mathrm{LAV}_{\min }\right)$, maximal $\left(\mathrm{LAV}_{\max }\right)$, and pre-atrial contraction volume $\left(\mathrm{LAV}_{\text {pre-c }}\right) \cdot \mathrm{LAV}_{\text {pre-c }}$ was defined as the last phase prior to atrial contraction. From these volumes, the total LA emptying fraction (LAEF) $\left(\left(\mathrm{LAV}_{\max }-\mathrm{LAV}_{\min }\right) \times 100 / \mathrm{LAV}_{\max }\right)$, passive LAEF $\left(\left(\mathrm{LAV}_{\max }-\mathrm{LAV}_{\text {pre-c }}\right) \times 100 / \mathrm{LAV}_{\max }\right)$, and active LAEF $\left(\left(\mathrm{LAV}_{\text {pre-c }}-\mathrm{LAV}_{\text {min }}\right) \times 100 / \mathrm{LAV}_{\text {pre-c }}\right)$ were derived. LAV index maximum $\left(\mathrm{LAVI}_{\max }\right)$ was calculated by dividing $\mathrm{LAV}_{\max }$ by body surface area.

\section{LA strain assessment}

Longitudinal LA strain analysis was performed using the feature tracking module in cvi42 (Circle Cardiovascular Imaging, Inc.). Endocardial and epicardial contours of the LA were traced in the end-diastolic phase of the long-axis two-chamber and four-chamber cine images. The automatic contour tracking algorithm was used and manual adjustments were applied, if necessary. This algorithm places a set of control points on the middle curve of the myocardial wall in the reference phase based on 
the drawn endocardial and epicardial contours. Subsequently, the position of the control points are detected based on the feature tracked boundaries in all the other phases to calculate longitudinal displacement.

Longitudinal strain measurements were subdivided into LA reservoir strain, conduit strain and contractile strain [14]. The time period (duration) for each phase was also calculated. Furthermore, LA peak positive strain rate, LA peak early negative strain rate, and LA peak late negative strain rate were measured using longitudinal strain rate curves. An illustration of LA strain analysis during the cardiac cycle is shown in Fig. 1 including a representative example of LA endocardial and epicardial contours.

\section{Right atrial volume and strain assessment}

Volumetric data of the right atrium (RA) were derived from the four-chamber cine images. RA volumes (RAV) (minimal $\left(\mathrm{RAV}_{\min }\right)$, maximal $\left(\mathrm{RAV}_{\max }\right)$, and pre-atrial contraction volume $\left(\mathrm{RAV}_{\text {pre-c }}\right)$ as well as the total $\mathrm{RA}$ emptying fraction (RAEF), passive RAEF, and active RAEF were calculated. RAV index maximum $\left(\right.$ RAVI $\left._{\max }\right)$ was calculated by dividing $\mathrm{RAV}_{\text {max }}$ by body surface area. Phasic RA longitudinal strain analysis was performed on the four-chamber cine images analogous to LA strain analysis as described above. A visual representation of the RA strain analysis is presented in the supplements (Additional file 1: Figure S1).

\section{LA fibrosis and sphericity assessment}

Quantification of LA fibrosis and calculation of LA sphericity was performed using open source software (CEMRG (Cardiac Electro-Mechanics Research Group), King's College London, United Kingdom) [15]. The LA blood pool including pulmonary vein extensions was segmented semi automatically in the 3D CE-MRA images on axial slices using a thresholding tool. The interpolated contours were adjusted manually in each axial plane. A 2 voxel $(1.25 \mathrm{~mm})$ surface dilation was used to define the epicardial border. Thereafter, the 3D CE-MRA images were co-registered with the 3D LGE images. A 3D reconstruction of the LA was generated and the LA appendage and the pulmonary veins were excluded at their ostia defined as the point of deflection from the LA wall. The mitral valve annulus was used to separate the LA from the LV. Signal intensity was normalized to the mean blood pool intensity according to the image intensity ratio method [16]. LA LGE was calculated using a default image intensity ratio threshold of $1.2[17,18]$, and was reported as percentage of the total LA surface.

In addition, the 3D LA shell obtained in CE-MRG was used to calculate LA sphericity using the algorithms published by Bisbal and colleagues [19]. In this regard, a LA sphericity of $100 \%$ represents a perfect sphere, whereas atria with a non-spherical shape will have a lower value.

\section{Reproducibility}

Inter- and intraobserver variability for the LA strain analysis was assessed in 15 patients (by L.H. and A.D.). Reproducibility of LA LGE quantification was assessed in 15 patients (by L.H. and P.B.).

\section{Statistical analysis}

Data are presented as mean \pm standard deviation (SD) for normally distributed data and median including interquartile range (IQR) for data with a non-normal distribution. Normality of continuous data was assessed by inspection of histograms and Q-Q plots. To test for differences between two groups the Student $t$-test or Mann-Whitney $U$ test was used, as appropriate. Pearson's correlation was used to quantify associations between continuous variables. Intra- and inter-observer variability of LA strain measurements were assessed by intraclass correlation coefficients (ICC) for absolute agreement based on two-way random model. Data were considered significant if $P$-value $<0.05$. Statistical analysis was performed using SPSS Statistics (version 26, Statistical Package for the Social Sciences, International Business Machines, Inc., Armonk, New York, USA).

\section{Results}

\section{Patient characteristics}

Good quality cine images were available in $90 \%$ of $\mathrm{AF}$ patients (94/105). Assessable 3D CE-MRA and 3D LGE images for quantification of LA fibrosis were available in 82 patients $(78 \%)$. The baseline characteristics of the study population are presented in Table 1 . In the AF group $(n=94)$, mean age was $60 \pm 9$ years and $64 \%$ were male. The study cohort consisted of $62(66 \%)$ patients with paroxysmal AF and 32 (34\%) patients with persistent $\mathrm{AF}$. The median duration between $\mathrm{AF}$ diagnosis and CMR scan was 32 months (14-83 months). In the healthy control group $(n=19)$, mean age was $58 \pm 4$ years and $58 \%$ were male, which was comparable to the AF group.

\section{LA volume in AF patients and controls}

LA volumetric parameters are summarized in Table 2 . LAVI $_{\max }$ was significantly higher in AF patients $\left(49 \pm 15 \mathrm{ml} / \mathrm{m}^{2}\right.$ vs. $37 \pm 8 \mathrm{ml} / \mathrm{m}^{2}$ in controls; $\left.P<0.01\right)$. Also, a lower LAEF was observed in the AF patients ( $52 \pm 13 \%$ vs. $64 \pm 8 \%$ in healthy controls, $P<0.001$ ). 


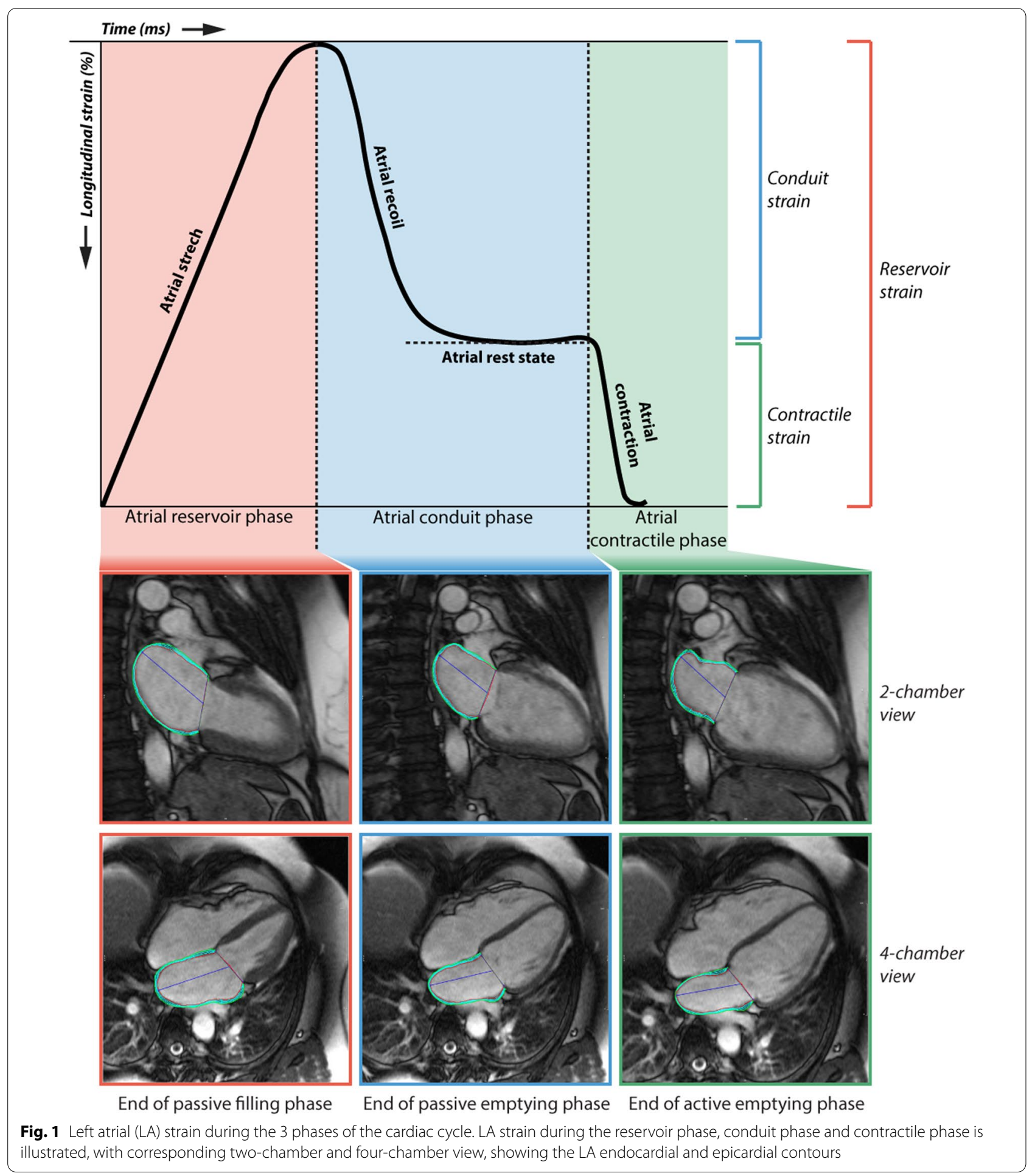

\section{LA reservoir, conduit and contractile strain in AF patients} and controls

In comparison with controls, all measures of phasic strain, i.e. reservoir strain, conduit strain, and contractile strain, were impaired in AF patients $(-15.9 \pm 3.8 \%$ vs. $-21.1 \pm 3.6 \% P<0.001,-8.7 \pm 2.7 \%$ vs. $-12.6 \pm 2.5 \%$ $P<0.001$ and $-7.2 \pm 2.3 \%$ vs. $-8.6 \pm 2.2 \% P=0.02$, respectively; Fig. 2). LA conduit strain time was significantly different between AF patients $(430 \pm 131 \mathrm{~ms}$ vs. $354 \pm 101 \mathrm{~ms}, P=0.02$ ) while LA reservoir strain time 
Table 1 Baseline characteristics of the study population

\begin{tabular}{|c|c|c|c|}
\hline & AF patients $(n=94)$ & $\begin{array}{l}\text { Healthy } \\
\text { controls } \\
(n=19)\end{array}$ & $P$-value \\
\hline \multicolumn{4}{|l|}{ Demographics } \\
\hline Age, years & $60 \pm 9$ & $58 \pm 4$ & 0.21 \\
\hline Male gender & $60(64 \%)$ & $11(58 \%)$ & 0.63 \\
\hline Weight (kg) & $84 \pm 14$ & $80 \pm 12$ & 0.44 \\
\hline Height (cm) & $180 \pm 11$ & $176 \pm 8$ & 0.15 \\
\hline $\mathrm{BMI}\left(\mathrm{kg} / \mathrm{m}^{2}\right)$ & $25.9 \pm 3.4$ & $25.6 \pm 5.1$ & 0.84 \\
\hline BSA (Mosteller)* & $2.0 \pm 0.2$ & $2.0 \pm 0.2$ & 0.48 \\
\hline $\mathrm{CHA}_{2} \mathrm{DS}_{2}$-VASc score & $1.2 \pm 1.2$ & - & - \\
\hline Hypertension & $30(32 \%)$ & - & - \\
\hline Diabetes mellitus & $4(4 \%)$ & - & - \\
\hline \multicolumn{4}{|l|}{ Medications } \\
\hline ACE inhibitor or ARB & $29(30.9 \%)$ & - & - \\
\hline Spironolactone & $3(3.2 \%)$ & - & - \\
\hline Amiodarone & $10(11.0 \%)$ & - & - \\
\hline Anticoagulation & 77 (81.9\%) & - & - \\
\hline \multicolumn{4}{|l|}{ AF history } \\
\hline Paroxysmal AF & $62(66 \%)$ & - & - \\
\hline Persistent AF & $32(34 \%)$ & - & - \\
\hline $\begin{array}{l}\text { Time between AF } \\
\text { diagnosis and CMR } \\
\text { (months) }\end{array}$ & $32(14-83)$ & - & - \\
\hline
\end{tabular}

Values are expressed as number (percentage), mean \pm SD or median (25-75th percentile). $A C E$ angiotensin-converting-enzyme, $A R B$ Angiotensin-receptorblocker, $A F$ atrial fibrillation, $B M I$ body mass index, $B S A$ body surface area, $\mathrm{CHA}_{2} D \mathrm{~S}_{2}$ VASC history of congestive heart failure, hypertension, diabetes mellitus, stroke/transient ischemic attack/prior thromboembolism, vascular disease, age and sex, CMR cardiovascular magnetic resonance. * ${ }^{*}$ Calculated by the Mosteller method ((height $(\mathrm{cm}) \times$ weight $\left.(\mathrm{kg}) / 3600)^{1 / 2}\right)$

and LA contractile strain time were similar (Table 2). LA phasic strain was significantly associated with LAVI $_{\text {max }}$ (reservoir strain; $r=-0.48, P<0.001$, conduit strain; $\mathrm{r}=-0.33, P<0.01$, contractile strain; $\mathrm{r}=-0.39$, $P<0.001)$.

\section{RA remodeling as compared to $\mathrm{LA}$ remodeling in $\mathrm{AF}$ patients}

Analysis of RA parameters could not be performed in 14 patients. $\mathrm{RAVI}_{\max }$ was similar among AF patients and healthy controls $\left(49 \pm 16 \mathrm{ml} / \mathrm{m}^{2}\right.$ vs. $45 \pm 11 \mathrm{ml} / \mathrm{m}^{2}$, $P=0.52)$ while RAEF was different between these two groups ( $45 \pm 10 \%$ vs. $52 \pm 11 \%, P<0.01$, respectively). RA strain parameters were not different between AF patients and healthy controls (Additional file 1: Table S1). In AF patients, $\mathrm{RAVI}_{\max }$ and $\mathrm{LAVI}_{\max }$ had a weak but significant correlation $(r=0.33, P<0.01)$ while RAEF and LAEF had no significant correlation $(r=0.21, P=0.07)$. RA reservoir strain was correlated with LA reservoir strain $(\mathrm{r}=0.36, P<0.01)$ (Additional file 1: Figure S2).
Impaired LA reservoir and conduit strain in AF patients with extensive fibrosis

To gain insight into the association between LA fibrosis and phasic strain, AF patients were dichotomized into groups according to the median percentage of LA LGE (low LGE $\leq 25.3 \%$ and high LGE > 25.3\%) (Table 3). Age, LA volumes, and LA sphericity were comparable between AF patients with a low and high degree of LA LGE. Patients with hypertension were more often classified in the high degree LA LGE group (high LGE: 44\% vs. low LGE: $22 \%, P=0.03$ ). Passive LAEF was significantly lower in patients with a high degree of LA LGE ( $24 \pm 9 \%$ vs. $29 \pm 11 \%, P=0.03)$. Passive strain parameters, i.e. LA reservoir and conduit strain, were also significantly impaired in patients with a high degree of LA LGE $(-15.0 \pm 3.9 \%$ vs. $-16.9 \pm 3.3 \%, P=0.02$ and $-7.9 \pm 2.7 \%$ vs. $-9.5 \pm 2.6 \%, P=0.01$, respectively). Contractile strain however, was comparable between patients with high and low LGE $(-7.1 \pm 2.3 \%$ vs. $-7.4 \pm 2.4 \%, P=0.55$; Table 3 , Fig. 3 ). LA conduit function showed the closest correlation to the extent of LA fibrosis (LA conduit strain Pearson correlation coefficient $(\mathrm{r})=-0.33, P<0.01$; LA reservoir strain $\mathrm{r}=-0.29, P<0.01 ;$ LA contractile strain $\mathrm{r}=-0.07$, $P=0.53)$.

\section{Paroxysmal AF versus persistent AF}

Finally, we compared parameters of LA and RA remodeling in patients with paroxysmal and persistent AF. Within the AF group, a higher maximal LAV and RAV was found in patients with persistent AF in comparison to patients with paroxysmal AF $\left(\mathrm{LAV}_{\text {max }}: 113 \pm 33 \mathrm{ml}\right.$ vs. $94 \pm 30 \mathrm{ml}, P=0.02, \mathrm{RAV}_{\max }: 110 \pm 37 \mathrm{ml}$ vs. $92 \pm 32 \mathrm{ml}$, $P=0.03)$. No difference was found for LA sphericity $(P=0.52)$. Although LAEF and LA strain values tended to be lower in patients with persistent AF, no significant difference between the two groups was found. Finally, no difference was found in the extent of LA LGE between patients with paroxysmal AF and persistent AF $(28.0 \pm 13.8 \%$ and $23.9 \pm 19.4 \%, P=0.30)$ (Additional file 1: Table S2, Figure S3).

\section{Reproducibility}

Fifteen randomly selected patients underwent repeated review to assess intra- and inter-observer reliability. The ICC for inter-reader variability of LA strain measurements was 0.85 (95\% confidence interval: 0.73-0.91; L.H., A.D.). The ICC for intra-reader variability of LA strain measurements was 0.90 (95\% confidence interval: $0.83-$ 0.95). For LA LGE analysis, the ICC for inter-reader variability was 0.93 (95\% confidence interval: 0.81-0.97; L.H., 
Table 2 CMR characteristics of the study population

\begin{tabular}{|c|c|c|c|}
\hline $\mathrm{i}$ & AF patients $(n=94)$ & Healthy Controls $(n=19)$ & $P$-value \\
\hline \multicolumn{4}{|l|}{ LA volume } \\
\hline LA volume - $\min (\mathrm{ml})$ & $50 \pm 28$ & $26 \pm 11$ & $<0.001$ \\
\hline LA volume - max (ml) & $100 \pm 32$ & $70 \pm 15$ & $<0.001$ \\
\hline LA volume index- $\max \left(\mathrm{ml} / \mathrm{m}^{2}\right)$ & $49 \pm 15$ & $37 \pm 8$ & $<0.01$ \\
\hline LA sphericity (\%)* & $79.5 \pm 3.0$ & & \\
\hline \multicolumn{4}{|l|}{ LA function volumetric } \\
\hline Total LAEF (\%) & $52 \pm 13$ & $64 \pm 8$ & $<0.001$ \\
\hline Passive LAEF (\%) & $27 \pm 10$ & $33 \pm 8$ & $<0.01$ \\
\hline Active LAEF (\%) & $25 \pm 9$ & $30 \pm 7$ & 0.03 \\
\hline \multicolumn{4}{|l|}{ LA strain } \\
\hline LA reservoir strain (\%) & $-15.9 \pm 3.8$ & $-21.1 \pm 3.6$ & $<0.001$ \\
\hline LA conduit strain (\%) & $-8.7 \pm 2.7$ & $-12.6 \pm 2.5$ & $<0.001$ \\
\hline LA contractile strain (\%) & $-7.2 \pm 2.3$ & $-8.6 \pm 2.2$ & 0.02 \\
\hline LA peak positive strain rate & $0.72 \pm 0.24$ & $0.87 \pm 0.16$ & $<0.01$ \\
\hline LA peak early negative strain rate & $-0.82 \pm 0.33$ & $-1.22 \pm 0.30$ & $<0.001$ \\
\hline LA peak late negative strain rate & $-0.83 \pm 0.30$ & $-0.97 \pm 0.26$ & 0.5 \\
\hline LA reservoir strain time (ms) & $392 \pm 45$ & $383 \pm 35$ & 0.39 \\
\hline LA conduit strain time (ms) & $430 \pm 131$ & $354 \pm 101$ & 0.02 \\
\hline LA contractile strain time (ms) & $137 \pm 41$ & $123 \pm 25$ & 0.15 \\
\hline LA LGE $(n=82)(\%)^{*}$ & $26.56 \pm 16.0$ & & \\
\hline \multicolumn{4}{|l|}{ LV parameters } \\
\hline LV ESV (ml) & $69 \pm 22$ & $55 \pm 12$ & $<0.01$ \\
\hline LV EDV (ml) & $168 \pm 42$ & $146 \pm 27$ & 0.03 \\
\hline LVEF (\%) & $59 \pm 7$ & $62 \pm 5$ & 0.08 \\
\hline
\end{tabular}

Values are expressed as mean $\pm S D$. $A F$ atrial fibrillation, $b p m$ beats per minute, $C M R$ cardiovascular magnetic resonance imaging, $E D V$ end diastolic volume, $E F$ ejection fraction, ESV end systolic volume, $L A$ left atrial; $L A E F$ left atrial emptying fraction, $L G E$ late gadolinium enhancement, $L V$ left ventricular, $L V E F$ left ventricular ejection fraction. Bold values denote statistical significance at the $P<0.05$ level

*Not obtained in healthy volunteers due to the contrast agent dependency of the acquisition
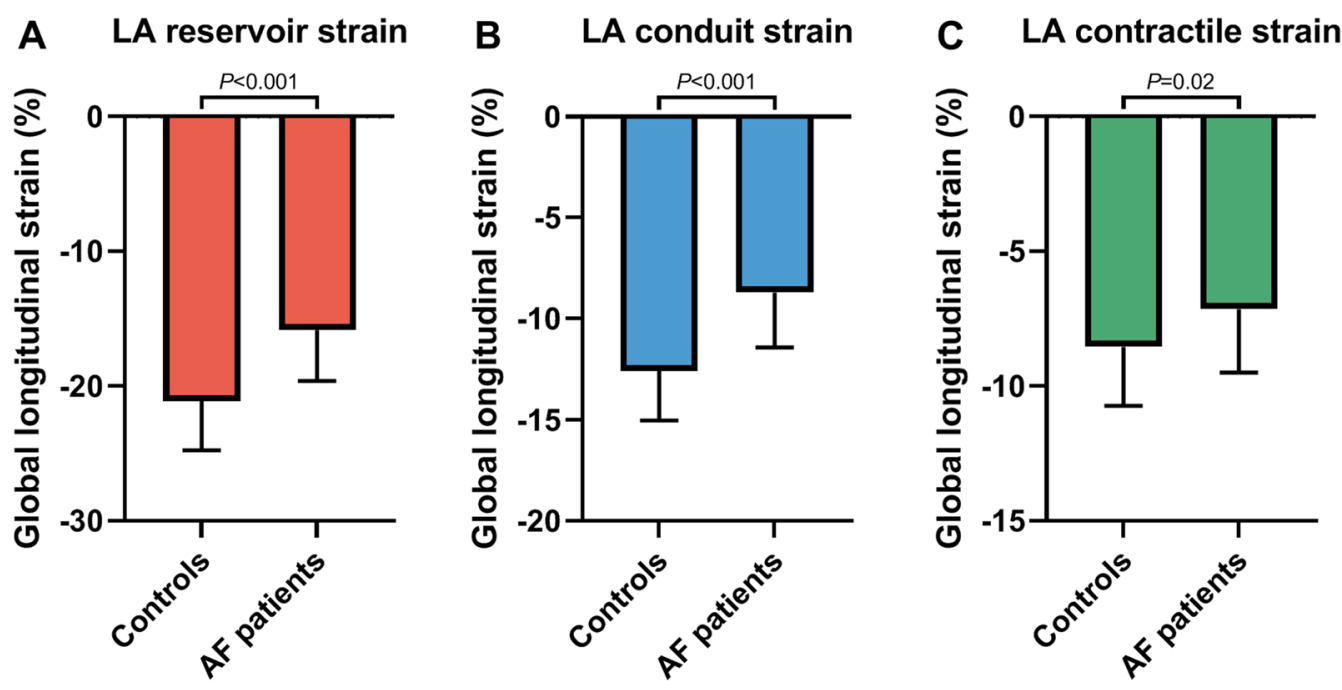

Fig. 2 LA phasic strain in atrial fibrillation patients and healthy controls. A $L A$ reservoir strain, $\mathbf{B} L A$ conduit strain and $\mathbf{C} L A$ contractile strain are impaired in patients with atrial fibrillation (AF), as compared to healthy controls. AF atrial fibrillation, LA left atrial. Data are presented as bars with mean and SD 
Table 3 Patient characteristics stratified according to low and high extent of LGE

\begin{tabular}{|c|c|c|c|}
\hline & Low $(\leq 25.3 \%)$ LGE $(n=41)$ & High $(>25.3 \%)$ LGE $(n=41)$ & $P$-value \\
\hline Age, years & $59 \pm 10$ & $61 \pm 7$ & 0.42 \\
\hline BSA (Mosteller)* & $2.1 \pm 0.2$ & $2.0 \pm 0.2$ & 0.15 \\
\hline CHA2DS2-VASc score & $1.1 \pm 1.2$ & $1.3 \pm 1.1$ & 0.50 \\
\hline Hypertension & $9(22 \%)$ & $18(44 \%)$ & 0.03 \\
\hline Diabetes mellitus & $2(5 \%)$ & $2(5 \%)$ & 1.00 \\
\hline LA volume index-max (ml/m2) & $48 \pm 12$ & $51 \pm 16$ & 0.36 \\
\hline LA sphericity (\%) & $79.7 \pm 2.8$ & $79.3 \pm 3.3$ & 0.61 \\
\hline \multicolumn{4}{|l|}{ LA function volumetric } \\
\hline Total LAEF (\%) & $55 \pm 11$ & $50 \pm 15$ & 0.09 \\
\hline Passive LAEF (\%) & $29 \pm 11$ & $24 \pm 9$ & 0.03 \\
\hline Active LAEF (\%) & $25 \pm 9$ & $25 \pm 11$ & 0.98 \\
\hline \multicolumn{4}{|l|}{ LA strain } \\
\hline LA reservoir strain (\%) & $-16.9 \pm 3.3$ & $-15.0 \pm 3.9$ & 0.02 \\
\hline LA conduit strain (\%) & $-9.5 \pm 2.6$ & $-7.9 \pm 2.7$ & 0.01 \\
\hline LA contractile strain (\%) & $-7.4 \pm 2.4$ & $-7.1 \pm 2.3$ & 0.55 \\
\hline LA peak positive strain rate & $0.76 \pm 0.27$ & $0.70 \pm 0.22$ & 0.36 \\
\hline LA peak early negative strain rate & $-0.89 \pm 0.31$ & $-0.76 \pm 0.35$ & 0.08 \\
\hline LA peak late negative strain rate & $-0.88 \pm 0.28$ & $-0.80 \pm 0.33$ & 0.22 \\
\hline LA reservoir strain time (ms) & $396 \pm 51$ & $383 \pm 41$ & 0.22 \\
\hline LA conduit strain time (ms) & $452 \pm 133$ & $410 \pm 130$ & 0.15 \\
\hline LA contractile strain time (ms) & $137 \pm 35$ & $132 \pm 31$ & 0.51 \\
\hline
\end{tabular}

Values are expressed as number (percentage) or mean \pm SD. BMI body mass index, BSA body surface area, CHA2DS2VASC history of congestive heart failure, hypertension, diabetes mellitus, stroke/transient ischemic attack/prior thromboembolism, vascular disease, age and sex, LA left atrium, LAEF left atrial emptying fraction, $L G E$ late gadolinium enhancement. Bold values denote statistical significance at the $P<0.05$ level

${ }^{*}$ Calculated by the Mosteller method ((height $(\mathrm{cm}) \times$ weight $\left.\left.(\mathrm{kg}) / 3600\right)^{1 / 2}\right)$
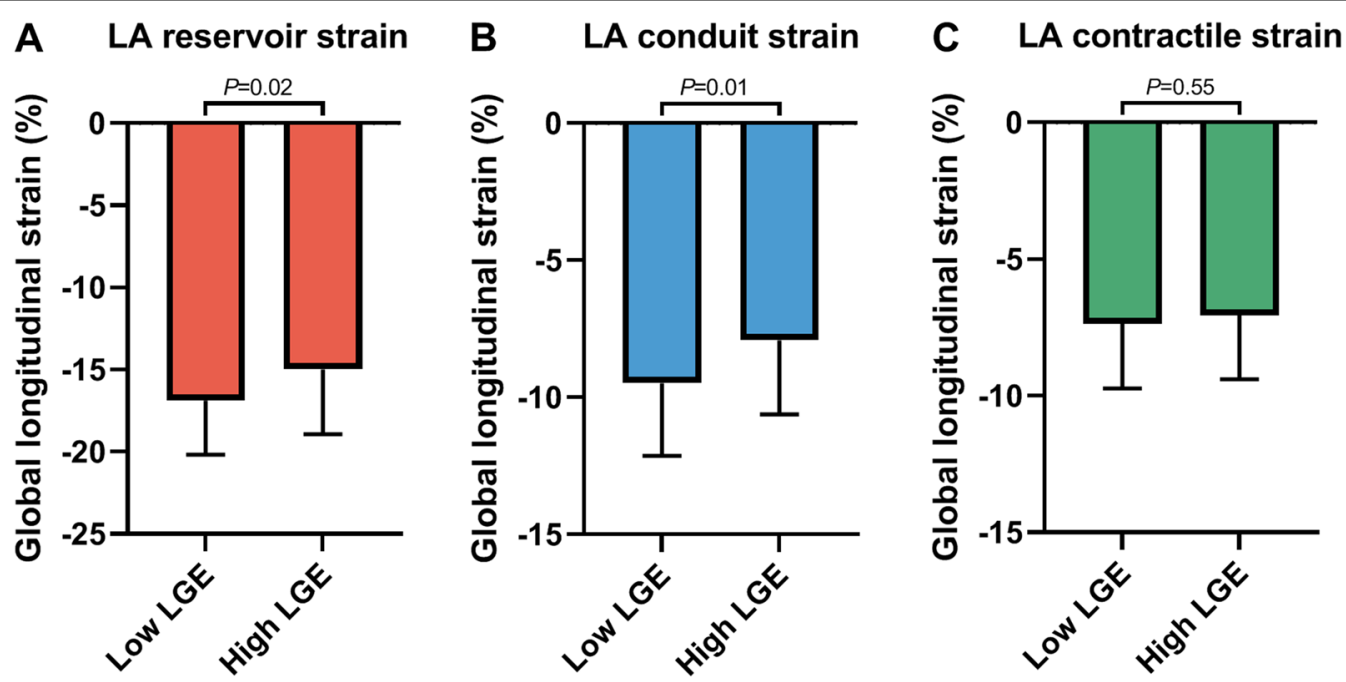

Fig. 3 The association between the extent of $L A$ late gadolinium enhancement and phasic $L A$ strain in $A F$ patients. $\mathbf{A} L A$ reservoir strain, $\mathbf{B} L A$ conduit strain and $C L A$ contractile strain, stratified according to low and high degree of $L A$ late gadolinium enhancement (LGE) in $A F$ patients are depicted. LA left atrial, LGE late gadolinium enhancement. Data are presented as bars with mean and SD 
P.B.) and the ICC for intra-reader variability was 0.96 (95\% confidence interval: 0.87-0.99).

\section{Discussion}

Taking advantage of state-of-the-art CMR feature tracking to assess atrial strain, we demonstrated that all components of LA strain, i.e. LA reservoir, LA conduit, and LA contractile strain, are markedly depressed in AF patients as compared to healthy controls. A prolonged LA conduit strain time was found in AF patients compared to controls. Furthermore, we found lower LA reservoir strain and LA conduit in AF patients with extensive LA fibrosis. Interestingly, LA contractile strain was comparable between AF patients with low and high extent of fibrosis.

In the last decades, LA remodeling has been recognized as an important prognostic marker in AF [20]. AF induces a vicious circle of structural and functional remodeling that in turn instigates AF recurrence, which can ultimately become irreversible [21]. Timely interventions may decelerate and perhaps reverse this pathophysiologic process and improve clinical outcome. In this regard, detailed characterization of atrial remodeling, and understanding of the interplay between structural remodeling and function is essential.

It has been suggested that alterations in LA strain may precede the structural changes associated with LA remodeling [22, 23], making LA strain an interesting tool to monitor this process. Also, comprehensive assessment of all three functional phases of the LA may provide additional insight into LA remodeling, in addition to structural parameters such as LA volume, geometry and fibrosis solely.

In the present study, all components of LA strain i.e. LA reservoir, LA conduit and LA contractile strain, were impaired in the AF patients as compared to healthy controls, while all AF patients were in sinus rhythm during CMR. Also, a prolonged LA conduit strain time period was observed in AF patients which may be an expression of the enlarged and more ridged LA. The impaired phasic function was associated with a larger LA volume. Furthermore, a larger LA volume was found in patients with persistent $\mathrm{AF}$, as compared to paroxysmal AF, which may be explained by a more advanced state of LA remodeling [10, 22]. Interestingly, there was no difference in LA volumetric function, LA strain, and LA fibrosis between patients with paroxysmal AF and persistent AF. A plausible explanation could be that persistent AF patients in our study cohort had either a mild form of persistent AF as all patients were in sinus rhythm during the scan. Moreover, the median time between AF diagnosis and the CMR scan was similar between patients with paroxysmal AF and persistent AF (paroxysmal AF: 35 months, persistent AF: 27 months, $P=0.75$ ) suggesting that these patients may have a rather corresponding LA remodeling stage. Additionally, the clinical value of phasic LA strain in relation to conventional LA remodeling parameters for predicting recurrent $\mathrm{AF}$ after catheter ablation has yet to be established and hence will be studied in future research.

LA wall fibrosis is a hallmark of structural remodeling in patients with $\mathrm{AF}$, and hence, might contribute to an impaired LA function $[4,17,24]$. Habibi et al. quantified LA fibrosis in AF patients and reported lower LA strain rates in the patients with a high degree of fibrosis [10]. Our results for the first time show lower LA reservoir strain and LA conduit strain in AF patients with more extensive LA fibrosis. Of interest, no association was found for contractile strain. Experimental studies have shown that atrial remodeling in AF is characterized by the presence of predominantly interstitial fibrosis and not by replacement fibrosis [25]. This interstitial fibrosis causes regional variation in myofiber architecture, which may impact atrial compliance, crucial for proper LA reservoir and LA conduit function [26, 27]. Likewise, interstitial fibrosis may impair LA reservoir and conduit strain, whereas active LA contractile function remains largely unaffected as cardiomyocytes are not replaced by fibrosis. This observation is supported in previous findings by Chesc et al. demonstrating a lack of association between active LAEF and the amount of LA fibrosis [28]. Structural alterations of the myocardium may have a greater effect on passive LA function while the LA contractile function may serve as a compensatory mechanism to maintain proper LV filling. As a results, contractile strain may not be different between patients with a high and low degree of LA fibrosis.

We found that AF is associated with structural and functional LA changes. Interestingly, minimal RA volume and RAEF were also different between healthy subjects and AF patients indicating a certain extent of RA involvement in AF. This bi-atrial remodeling was not characterized by a difference in RA strain between healthy subjects and AF patients. Literature on the relationship between RA remodeling and LA remodeling in AF patients is limited. A study by Xie et al. found that higher RA volume indices were independently associated with incident AF in a model adjusted for demographics and traditional risk factors while RAEF and RA global strain were not [29]. Moreover, the presence and impact of RA fibrosis detected by LGE-CMR in AF patients on RA function remains unknown and hence would be of interest for future studies. 


\section{Limitations}

Our study has several limitations. Firstly, there are multiple methods described for the detection of LA fibrosis using LGE-CMR [30]. In the present study, the IIR method was chosen to assess atrial fibrosis as this method is proposed to be a more consistent and reproducible method compared to algorithms relying on a certain number of standard deviations over a reference signal intensity. However, there is currently no complete agreement on IIR thresholds for LA LGE since direct histological validation on specific IIR cut-off values is lacking [31]. Based on recent literature, an IIR cut-off value of 1.2 was chosen to define atrial fibrosis [17]. Moreover, LA fibrosis quantification could not be performed in healthy subjects since the contrast agent based acquisition was not performed in this group. Therefore, no comparison could be made between the healthy control group and AF group in terms of LA LGE.

Secondly, the LA wall is thin $(\approx 2 \mathrm{~mm})$ and segmentation of the LA wall may be cumbersome in LGE-CMR images. Considering the thin atrial wall relative to the LGE-CMR voxel size, the CMR LA wall signal is subject to partial volume effects. Therefore, structures adjacent to the atrial wall such as the descending aorta may influence fibrosis quantification in this specific area. Moreover, the potential presence of inflammation and edema in the LA wall may have influenced the quantification of LA fibrosis using LGE [32, 33].

Thirdly, according to the EHRA AF guidelines, AF classification into either paroxysmal or persistent AF was based on the more common AF type the last six months. The patients classified as persistent AF patients in our study cohort were in sinus rhythm during the CMR scan and therefore may represent a selected category of persistent AF patients, having a mild form of persistent AF. This could explain the minimal differences in LA remodeling parameters between the two AF types. AF patients with ongoing persistent AF, often with a more advanced state of LA remodeling, were excluded from the study.

Lastly, because of LV/LA focused cine imaging, the RA could not be analyzed in a subset of patients. Moreover, RA volumes and strain were calculated only from the four-chamber cine images, while LA volumes and strain were calculated from two-chamber and four-chamber cine images using the biplane method. Assessment of the presence and impact of RA fibrosis in AF patients was not performed in this study and will be subject of future research.

\section{Conclusions}

This study further establishes LA strain as marker of LA remodeling in AF patients, and for the first time demonstrates an important association between impaired (passive) LA reservoir strain and conduit strain in AF patients with extensive LA fibrosis. The amount of LA fibrosis, however, did not affect LA contractile strain. Future studies are required to study the biologic nature of this association and possible prognostic and therapeutic implications.

\begin{abstract}
Abbreviations
3D: Three-dimensional; AF: Atrial fibrillation; CE-MRA: Contrast enhanced magnetic resonance angiography; CMR: Cardiovascular magnetic resonance; ECG: Electrocardiogram; EDV: End-diastolic volume; ESV: End-systolic volume; LA: Left atrium/left atrial; LAEF: Left atrial emptying fraction; LAV: Left atrial volume; LAVi: Left atrial volume index; LGE: Late gadolinium enhancement; LV: Left ventricle/left ventricular; LVEF: Left ventricular ejection fraction; PVI: Pulmonary vein isolation; RA: Right atrium/right atrial; RAEF: Right atrial emptying fraction; RAV: Right atrial volume; RAVi: Right atrial volume index.
\end{abstract}

\section{Supplementary Information}

The online version contains supplementary material available at https://doi. org/10.1186/s12968-021-00820-6.

Additional file 1: Figure S1. Right atrial feature tracking strain contours. Figure S2. Right atrial volumes and strain vs. left atrial volumes and strain in AF patients. Figure S3. LA volume and function in patients with paroxysmal and persistent AF. Table S1. Right atrial parameters of the study population. Table S2. CMR characteristics in patients with paroxysmal and persistent AF.

\section{Acknowledgements}

None.

\section{Authors' contributions}

$M G, C A$, and $A R$ were involved in the conception and design of the study. LH drafted the first version of the manuscript. MG, AL, PB, and MM were involved in the interpretation of data, as well as revising the manuscript for critically intellectual content. AD performed strain analysis at the images. All authors read, critically revised and approved the manuscript. All authors agree to be accountable for all aspects of the work in ensuring that questions related to the accuracy or integrity of any part of the work are appropriately investigated and resolved. This manuscript is not under consideration for publication elsewhere. None of the manuscript's contents have been previously published. All authors read and approved the final manuscript.

\section{Funding}

None.

\section{Availability of data and materials}

The datasets used and/or analyzed during the current study are available from the corresponding author upon reasonable request.

\section{Declarations}

\section{Ethics approval and consent to participate}

The study protocol was approved by the local medical ethics committee (Amsterdam UMC, location VU University Medical Center, Amsterdam, The Netherlands). Written informed consent was obtained from all individuals included in the study.

\section{Consent for publication}

Not applicable.

\section{Competing interests}

Dr. Götte is consultant for Circle Cardiovascular Imaging. All other authors have nothing to disclose. 
Received: 18 January 2021 Accepted: 11 October 2021

Published online: 11 November 2021

\section{References}

1. Iwasaki YK, Nishida K, Kato T, Nattel S. Atrial fibrillation pathophysiology: implications for management. Circulation. 2011;124(20):2264-74.

2. Al Ghamdi B, Hassan W. Atrial remodeling and atrial fibrillation: mechanistic interactions and clinical implications. J Atr Fibrillation . 2009;2(1):125.

3. Oakes RS, Badger TJ, Kholmovski EG, Akoum N, Burgon NS, Fish EN, et al. Detection and quantification of left atrial structural remodeling with delayed-enhancement magnetic resonance imaging in patients with atrial fibrillation. Circulation. 2009;119(13):1758-67.

4. Marrouche NF, Wilber D, Hindricks G, Jais P, Akoum N, Marchlinski F, et al. Association of atrial tissue fibrosis identified by delayed enhancement MRI and atrial fibrillation catheter ablation: the DECAAF study. JAMA. 2014:311(5):498-506.

5. Barbier P, Solomon SB, Schiller NB, Glantz SA. Left atrial relaxation and left ventricular systolic function determine left atrial reservoir function. Circulation. 1999;100(4):427-36.

6. Toma Y, Matsuda Y, Moritani K, Ogawa H, Matsuzaki M, Kusukawa R. Left atrial filling in normal human subjects: relation between left atrial contraction and left atrial early filling. Cardiovasc Res. 1987;21(4):255-9.

7. Manning WJ, Katz SE, Douglas PS, Silverman DI. Atrial ejection force: A noninvasive assessment of atrial systolic function. J Am Coll Cardiol. 1993;22(1):221-5

8. Chirinos JA, Sardana M, Ansari B, Satija V, Kuriakose D, Edelstein I, et al. Left atrial phasic function by cardiac magnetic resonance feature tracking is a strong predictor of incident cardiovascular events. Circ Cardiovasc Imaging. 2018;11(12):e007512.

9. Rasmussen SMA, Olsen FJ, Jorgensen PG, Fritz-Hansen T, Jespersen T, Gislason $\mathrm{G}$, et al. Utility of left atrial strain for predicting atrial fibrillation following ischemic stroke. Int J Cardiovasc Imaging. 2019;35(9):1605-13.

10. Habibi M, Lima JAC, Khurram IM, Zimmerman SL, Zipunnikov V, Fukumoto $K$, et al. Association of left atrial function and left atrial enhancement in patients with atrial fibrillation: cardiac magnetic resonance study. Circ Cardiovasc Imaging. 2015;8(2):e002769.

11. Leung M, Abou R, van Rosendael PJ, van der Bijl P, van Wijngaarden SE, Regeer MV, et al. Relation of echocardiographic markers of left atrial fibrosis to atrial fibrillation burden. Am J Cardiol. 2018;122(4):584-91.

12. Hindricks G, Potpara T, Dagres N, Arbelo E, Bax JJ, Blomström-Lundqvist C, et al. 2020 ESC Guidelines for the diagnosis and management of atrial fibrillation developed in collaboration with the European Association for Cardio-Thoracic Surgery (EACTS): the task force for the diagnosis and management of atrial fibrillation of the European Society of Cardiology (ESC) Developed with the special contribution of the European Heart Rhythm Association (EHRA) of the ESC. Eur Heart J. 2020;42(5):373-498.

13. Calkins H, Kuck KH, Cappato R, Brugada J, Camm AJ, Chen S-A, et al. 2012 HRS/EHRA/ECAS expert consensus statement on catheter and surgical ablation of atrial fibrillation: recommendations for patient selection, procedural techniques, patient management and follow-up, definitions, endpoints, and research trial design: a report of the Heart Rhythm Society (HRS) task force on catheter and surgical ablation of atrial fibrillation. EP Europace. 2012;14(4):528-606.

14. Gan GCH, Ferkh A, Boyd A, Thomas L. Left atrial function: evaluation by strain analysis. Cardiovasc Diagn Ther . 2018;8(1):29-46.

15. Razeghi O, Solís-Lemus JA, Lee AWC, Karim R, Corrado C, Roney CH, et al CemrgApp: an interactive medical imaging application with image processing, computer vision, and machine learning toolkits for cardiovascular research. SoftwareX. 2020;12:100570.

16. Khurram IM, Beinart R, Zipunnikov V, Dewire J, Yarmohammadi H, Sasaki $T$, et al. Magnetic resonance image intensity ratio, a normalized measure to enable interpatient comparability of left atrial fibrosis. Heart Rhythm. 2014;11(1):85-92.

17. Benito EM, Carlosena-Remirez A, Guasch E, Prat-González S, Perea RJ, Figueras R, et al. Left atrial fibrosis quantification by late gadoliniumenhanced magnetic resonance: a new method to standardize the thresholds for reproducibility. EP Europace. 2016;19(8):1272-9.

18. Bertelsen L, Alarcón F, Andreasen L, Benito E, Olesen MS, Vejlstrup N, et al Verification of threshold for image intensity ratio analyses of late gadolinium enhancement magnetic resonance imaging of left atrial fibrosis in 1.5T scans. Int J Cardiovasc Imaging. 2020;36(3):513-20.

19. Bisbal F, Guiu E, Calvo N, Marin D, Berruezo A, Arbelo E, et al. Left atrial sphericity: a new method to assess atrial remodeling. Impact on the outcome of atrial fibrillation ablation. J Cardiovasc Electrophysiol. 2013. https://doi.org/10.1111/jce.12116.

20. Thomas L, Abhayaratna WP. Left atrial reverse remodeling: mechanisms, evaluation, and clinical significance. JACC. 2017;10(1):65-77.

21. Nattel S, Guasch E, Savelieva I, Cosio FG, Valverde I, Halperin JL, et al. Early management of atrial fibrillation to prevent cardiovascular complications. Eur Heart J. 2014;35(22):1448-56.

22. Kuppahally SS, Akoum N, Burgon NS, Badger TJ, Kholmovski EG, Vijayakumar $S$, et al. Left atrial strain and strain rate in patients with paroxysmal and persistent atrial fibrillation: relationship to left atrial structural remodeling detected by delayed-enhancement MRI. Circ Cardiovasc Imaging. 2010;3(3):231-9.

23. Jarasunas J, Aidietis A, Aidietiene S. Left atrial strain —an early marker of left ventricular diastolic dysfunction in patients with hypertension and paroxysmal atrial fibrillation. Cardiovasc Ultrasound. 2018;16(1):29.

24. Khurram IM, Habibi M, Gucuk Ipek E, Chrispin J, Yang E, Fukumoto K, et al. Left atrial LGE and arrhythmia recurrence following pulmonary vein isolation for paroxysmal and persistent AF. JACC. 2016;9(2):142-8.

25. Everett THt, Olgin JE. Atrial fibrosis and the mechanisms of atrial fibrillation. Heart Rhythm. 2007;4(3 Suppl):S24-7.

26. Smaill BH. Fibrosis, myofibroblasts, and atrial fibrillation. Circulation. 2015;8(2):256-7.

27. Hansen BJ, Zhao J, Fedorov WV. Fibrosis and atrial fibrillation: computerized and optical mapping: a view into the human atria at submillimeter resolution. JACC. 2017;3(6):531-46.

28. Csécs I, Yamaguchi T, Kheirkhahan M, Czimbalmos C, Fochler F, Kholmovski EG, et al. Left atrial functional and structural changes associated with ablation of atrial fibrillation — cardiac magnetic resonance study. Int J Cardiol. 2020;305:154-60.

29. Xie E, Yu R, Ambale-Venkatesh B, Bakhshi H, Heckbert SR, Soliman EZ, et al. Association of right atrial structure with incident atrial fibrillation: a longitudinal cohort cardiovascular magnetic resonance study from the Multi-Ethnic Study of Atherosclerosis (MESA). J Cardiovasc Magn Reson. 2020:22(1):36.

30. Pontecorboli G, Figueras i Ventura RM, Carlosena A, Benito E, Prat-Gonzales $\mathrm{S}$, Padeletti L, et al. Use of delayed-enhancement magnetic resonance imaging for fibrosis detection in the atria: a review. EP Europace. 2016;19(2):180-9.

31. Sohns C, Marrouche NF. Atrial fibrillation and cardiac fibrosis. Eur Heart J. 2019;41(10):1123-31.

32. Mewton N, Liu CY, Croisille P, Bluemke D, Lima JAC. Assessment of myocardial fibrosis with cardiovascular magnetic resonance. J Am Coll Cardiol. 2011;57(8):891-903.

33. Bisbal F, Baranchuk A, Braunwald E, de Bayés Luna A, Bayés-Genís A. Atrial failure as a clinical entity. JACC. 2020;75(2):222-32.

\section{Publisher's Note}

Springer Nature remains neutral with regard to jurisdictional claims in published maps and institutional affiliations. 